FACTA UNIVERSITATIS

Series: Physical Education and Sport, Vol. 16, No 2, 2018, pp. 463 - 476

https://doi.org/10.22190/FUPES170116041V

Research article

\title{
THE EFECTS OF DIFFERENT SHOOTING PROGRAMS ON HANDGUN ACCURACY
}

\author{
UDC 799:311.2:351.811
}

\section{Goran Vučković ${ }^{1}$, Milivoj Dopsaj ${ }^{2}$, Bojan Mitrović ${ }^{3}$, Radivoje Janković ${ }^{1}$, Raša Dimitrijević ${ }^{1}$, Saša Mijalković ${ }^{1}$}

${ }^{1}$ Academy of Criminalistic and Police Studies, Belgrade, Serbia ${ }^{2}$ Faculty of Sport and Physical Education, University of Belgrade, Belgrade, Serbia ${ }^{3}$ Department for Police Education and Training, Ministry of Interior, Belgrade, Serbia

\begin{abstract}
Using a handgun is the one of the specialized physical abilities for police officers and it is an important part of the training programs in the Republic of Serbia police educational system. The constant monitoring of the achieved results is required. It is related both to the aspect of qualification for handling the pistol and to the aspect of improvement of training through reduction of the number of bullets and time necessary for planned programs. The aim of the study was to define the most efficient and most rational shooting training program which would enable improvement in the basic training for pistol handling. The sample consisted of 447 male students of the Academy of Criminalistic and Police Studies aged 19 to 22, divided into 7 groups, with each group following a different programme. All data were analyzed using descriptive statistics and the existence of a general difference of variability between the groups was determined by the ANOVA, while for the determination of partial difference between pairs of variables, the post hoc test with the application of Scheffe criterion was used. The results showed that there is a statistically significant difference in shooting accuracy among the groups $(F=28.840, p=0.000)$. Based on these results, there can be a reduction of costs of the existing training in the sense of the number of bullets and the time for training. Also, the data from this research could help in the implementation of a program which would ensure that the efficiency of shooting is the desired level.
\end{abstract}

Key words: Shooting, Pistol, Police, Training

Received January 16, 2017 / Accepted September 10, 2018

Corresponding author: Bojan Mitrović

Department for Police Education and Training, Železnička 22, 21208 Sremska Kamenica, Ministry of Interior, Belgrade, Serbia

Phone: +38121 462032• E-mail: bokimitrovic976@gmail.com 


\section{INTRODUCTION}

Practical training is organized to constantly improve the performance of police officers' security tasks, where one of the goals is the use of firearms (Morrison, 2005; Heim \& Schmidtbleicher, 2006). Current security risks are characterized by the use of firearms, both conventional (in cases of general crime, organised crime, terrorism, armed aggression, armed insurrection, civil war), and non-conventional (tendencies of terrorists - national extremists and religious fundamentalists and fanatics to come into possession of arms for mass destruction) (Vučković \& Dopsaj, 2007; Vučković, Dopsaj, Radovanović, \& Jovanović, 2008). There is greater probability that firearms may be used by the police during prevention and suppression of modern security threats (Kayihan, Ersöz, Özkan, \& Koz, 2013).

Despite intensive development, modern methods for the use of technical means failed to replace or eliminate the human factor in solving and implementing the most complex professional operations and tasks characteristic for the domain of policing (Roberg, 2004; Heim \& Schmidtbleicher, 2006). Since the human factor is still dominant in the police profession, the adequate education process is a very important prerequisite, both for efficient training of police officers from the aspect of adopting new motor skills (information) and their professional improvement, and for their efficient implementation within professional situations and tasks (Decker \& Huckabee, 2002; Vučković \& Dopsaj 2007; Vučković, Dopsaj, Radovanović, \& Jovanović, 2008; Kayihan, Ersöz, Özkan, \& Koz, 2013). The consequence is a real need for improved police training in firearms handling.

Issues in the basic training for using a pistol, monitoring and scientific-professional valorisation and control of the effects of the training processes represent the subject of research in all developed countries of the world (Vučković \& Dopsaj 2007, Vučković et al. 2008; Kayihan et al. 2013; Hech Dominski, Sousa Matias, Elpídio Cardoso, Brusque Crocetta, \& Andrade, 2015). Moreover, the subject of the research is the control and diagnostic procedure of all causalities in the system of policing (Decker \& Huckabee, 2002; White, 2002; Burke \& Mikkelsen, 2004; Morison, 2005; Heim \& Schmidtbleicher, 2006).

In previous programs of shooting training for police officers in the Republic of Serbia, the use of a pistol had either been reduced to a minimum, or was completely absent (Vučković, Dopsaj, \& Dujković, 2005). In cases of pistol handling, unlimited time for realisation of shooting was allowed, and the distance from the target was always the same. Such a way of realizing the shooting program is unspecific for the needs of police officers, it is reduced to the static completion of tasks and does not enable an adequate reaction in situations characteristic for the use of firearms in real situations (White, 2002; Morrison, 2005; Heim \& Schmidtbleicher, 2006; Vučković \& Dopsaj, 2007).

The approach of Serbia to the European Union has enabled the inclusion into new police officer firearms training programs. One of the first applied training programs for firearms handling was taken from the Organisation for Security and Cooperation in Europe (OSCE), and it comprised of 300 bullets and a shooting test of 60 bullets. This training program was applied in the educational system of the police of the Republic of Serbia, accomplished as a test for future police officers in qualifications for handling a pistol and as a form of shooting training of active police officers. Together with the increase of number of bullets for realisation of shooting, the OSCE training program is 
characterised by handling the pistol (drawing a pistol from the holster, loading a bullet into the firing chamber, re-inserting the magazine, putting the pistol back into the holster), time limitation (both for one bullet, two bullets, and for ten or fifteen bullets), and the constant change of distances, from three to fifteen meters (Vučković et al. 2005).

The implementation of the OSCE program has improved pistol training, but at the same time, it raised costs because of the increased number of bullets, number of days necessary for the realization of training and number of instructors. For that reason, it was necessary to define an optimally efficient program of training from the aspect of cost rationalization (cost benefit). However, rationalization of costs must not affect the quality of the training program. Also, the constant monitoring of the achieved training results and a regular check of the applied programs is required. It is related both to the aspect of qualifications for handling pistols and to the aspect of improvement of training through a reduction of the number of bullets and time necessary for planned programs. The goal of this paper is to define the most efficient and most rational shooting training program which would enable improvement in the basic training for pistol handling.

\section{METHODS}

\section{The sample of participants}

The sample consisted of the Academy of Criminalistic and Police Studies in Belgrade (ACPS) 447 male students, aged 19 to 22. They were divided into 7 groups, 80 participants were in the first group - Basic program (Prog_1), 57 participants in the second group (Prog_2), 44 participants in the third group (Prog_3), 56 participants in the fourth group (Prog_4), 62 participants in the fifth group (Prog_5), 74 participants in the sixth group (Prog_6), and 74 participants in the seventh group (Prog_7). All the participants had no previous knowledge in pistol handling and each group completed one different program of shooting training.

\section{Experimental-Training shooting program}

In order to assess the experimental-training programs, it was necessary to make programs that would follow the methodological principles of education, both related to handling firearms and distances, and especially for combinations of time limits for shooting, together with simultaneous changes of targets. 
Table 1 Characteristics of all programs

\begin{tabular}{|c|c|c|c|c|c|c|c|c|}
\hline$\dot{0}_{0}^{\infty}$ & $\begin{array}{l}\text { Number } \\
\text { of series } \\
\quad \text { (day } \\
\text { +night) }\end{array}$ & $\begin{array}{c}\text { Number } \\
\text { of bullets } \\
\text { (series) }\end{array}$ & Targets & $\begin{array}{c}\text { Distance } \\
(\mathrm{m})\end{array}$ & $\begin{array}{l}\text { Number } \\
\text { of } \\
\text { exercises }\end{array}$ & $\begin{array}{l}\text { Without/ } \\
\text { with } \\
\text { handling }\end{array}$ & $\begin{array}{l}\text { Without } \\
\text { /with } \\
\text { time }\end{array}$ & $\begin{array}{c}\text { Position } \\
\text { standing } \\
/ \\
\text { kneeling }\end{array}$ \\
\hline $\begin{array}{c}-7 \\
\text { ol } \\
0 \\
0 \\
0\end{array}$ & $\begin{array}{c}5 \\
(4+1)\end{array}$ & $\begin{array}{c}300 \\
(50+70 \\
+80+80 \\
+20)\end{array}$ & $\begin{array}{l}\text { "white paper" (in the first } \\
\text { series), circular with the } \\
\text { marked value of } 6 \text {, (in the } \\
\text { second series), silhouette - } \\
\text { only the value of } 10 \text { (the third } \\
\text { and fourth series), silhouette - } \\
\text { the whole silhouette } \\
\text { (for night shooting); }\end{array}$ & $\begin{array}{c}3,5,7,10 \\
\text { and } 15\end{array}$ & 28 & $10 / 18$ & $10 / 18$ & $23 / 5$ \\
\hline $\begin{array}{c}2 \\
N_{1} \\
01 \\
0 \\
0\end{array}$ & $\begin{array}{c}3 \\
(2+1)\end{array}$ & $\begin{array}{c}150 \\
(50+70 \\
+20)\end{array}$ & $\begin{array}{l}\text { "white paper" (in the first } \\
\text { series), silhouette - only the } \\
\text { value of } 10 \text { (in the second } \\
\text { series), silhouette - the whole } \\
\text { silhouette (for night shooting); }\end{array}$ & $\begin{array}{c}3,5,7,10 \\
\text { and } 15\end{array}$ & 15 & $8 / 7$ & $7 / 8$ & $11 / 4$ \\
\hline $\begin{array}{l}\text { m } \\
\text { ol } \\
0 \\
0 \\
0\end{array}$ & $\begin{array}{c}2 \\
(2+0)\end{array}$ & $\begin{array}{c}90 \\
(20+70)\end{array}$ & $\begin{array}{l}\text { Round - values from } 1 \text { - } 10 \text { (in } \\
\text { the first series), "white paper" } \\
\text { (for two exercises of the second } \\
\text { series), circular with the } \\
\text { marked value of } 6 \text {, (in the } \\
\text { third, fourth and fifth exercise } \\
\text { of the second series), silhouette } \\
\text { - only the value of } 10 \text { (in the } \\
\text { seventh and eighth exercise of } \\
\text { the second series) }\end{array}$ & $\begin{array}{l}3,5,7,10 \\
\text { and } 15\end{array}$ & 12 & $4 / 8$ & $9 / 3$ & $10 / 2$ \\
\hline $\begin{array}{l}v_{1} \\
01 \\
0 \\
0 \\
0\end{array}$ & $\begin{array}{c}2 \\
(1+1)\end{array}$ & $\begin{array}{c}80 \\
(60+20)\end{array}$ & $\begin{array}{l}\text { Silhouette - only the value of } \\
10 \text { (in the first series), } \\
\text { silhouette - the whole } \\
\text { silhouette (for night shooting) }\end{array}$ & $\begin{array}{l}3,5,7,10 \\
\text { and } 15\end{array}$ & 8 & $3 / 5$ & $1 / 7$ & $7 / 1$ \\
\hline $\begin{array}{l}n \\
01 \\
0 \\
0 \\
0\end{array}$ & $\begin{array}{c}2 \\
(2+0)\end{array}$ & $\begin{array}{c}90 \\
(40+50)\end{array}$ & $\begin{array}{l}\text { Circular with the marked value } \\
\text { of } 6 \text {, (in the first series), } \\
\text { silhouette - only the value of } \\
10 \text { (in the second series) }\end{array}$ & $\begin{array}{l}3,5,7,10 \\
\text { and } 15\end{array}$ & 9 & $3 / 6$ & $3 / 6$ & $8 / 1$ \\
\hline $\begin{array}{l}0 \\
01 \\
01 \\
0 \\
0\end{array}$ & $\begin{array}{c}3 \\
(3+0)+ \\
1 \text { (air) }\end{array}$ & $\begin{array}{c}205 \\
(50+70+ \\
85)+20 \\
\text { pellets }\end{array}$ & $\begin{array}{l}\text { Circular for the air rifle, "white } \\
\text { paper" (in the second series), } \\
\text { silhouette - only the value of } \\
10 \text { (in the third and fourth } \\
\text { series) }\end{array}$ & $\begin{array}{c}7,10,15 \\
\text { and } 20\end{array}$ & 22 & $21 / 1$ & $21 / 1$ & $22 / 0$ \\
\hline $\begin{array}{c}1 \\
01 \\
0 \\
0 \\
0\end{array}$ & $\begin{array}{c}5 \\
(5+0)+ \\
1 \text { (air) }\end{array}$ & $\begin{array}{c}325 \\
(50+70+ \\
85+60+6 \\
0)+20 \\
\text { pellets } \\
\end{array}$ & $\begin{array}{l}\text { Circular for the air rifle, "white } \\
\text { paper" (in the second series), } \\
\text { silhouette - only the value of } \\
10 \text { (in the third, fourth, fifth } \\
\text { and sixth series) }\end{array}$ & $\begin{array}{l}3,5,7,10 \\
15 \text { and } 20 \\
\text { meters }\end{array}$ & 34 & $23 / 11$ & $23 / 11$ & $32 / 2$ \\
\hline
\end{tabular}


Table 2 Characteristics of the Basic training program (Prog_1)

\begin{tabular}{|c|c|c|c|c|c|c|}
\hline Series & No. & Length & Loading & Positions & Realisation & Time \\
\hline \multirow{6}{*}{ I } & 1. & $5 \mathrm{~m}$ & $1 \times 10$ & Standing - preparatory & $10 \times 1$ bullet $^{1}$ & unlimited \\
\hline & 2. & $7 \mathrm{~m}$ & $1 \times 10$ & Standing - preparatory & $10 \times 1$ bullet & unlimited \\
\hline & 3. & $10 \mathrm{~m}$ & $1 \times 10$ & Standing - preparatory & $10 \times 1$ bullet & unlimited \\
\hline & 4. & $10 \mathrm{~m}$ & $1 \times 10$ & Standing - preparatory & $10 \times 1$ bullet & $5 \mathrm{~s}$ for one bullet \\
\hline & 5. & $10 \mathrm{~m}$ & $1 \times 5$ & Kneeling - preparatory & $5 \times 1$ bullet & unlimited \\
\hline & 6. & $10 \mathrm{~m}$ & $1 \times 5$ & Kneeling - preparatory & $5 \times 1$ bullet & unlimited \\
\hline \multirow{7}{*}{ II } & 1. & $7 \mathrm{~m}$ & $1 \times 10$ & Standing - preparatory & $10 \times 1$ bullet & unlimited \\
\hline & 2. & $5 \mathrm{~m}$ & $1 \times 10$ & Standing & $10 \times 1$ bullet & unlimited \\
\hline & 3. & $5 \mathrm{~m}$ & $1 \times 10$ & Standing & $10 \times 1$ bullet & unlimited \\
\hline & 4. & $7 \mathrm{~m}$ & $1 \times 10$ & Standing & $10 \times 1$ bullet & $8 \mathrm{~s}$ for one bullet \\
\hline & 5. & $10 \mathrm{~m}$ & $1 \times 10$ & Standing & $5 \times 2$ bullets & $8 \mathrm{~s}$ for two bullets \\
\hline & 6. & $15 \mathrm{~m}$ & $2 \times 5$ & Standing & $5 \times 2$ bullets & $8 \mathrm{~s}$ for two bullets \\
\hline & 7. & $15 \mathrm{~m}$ & $2 \times 5$ & Standing & $5 \times 2$ bullets & $8 \mathrm{~s}$ for two bullets \\
\hline \multirow{7}{*}{ III } & 1. & $15 \mathrm{~m}$ & $2 \times 10$ & Standing & $20 \times 1$ bullet & $8 \mathrm{~s}$ for one bullet \\
\hline & 2. & $10 \mathrm{~m}$ & $1 \times 5$ & Kneeling - preparatory & $1 \times 5$ bullets & unlimited \\
\hline & 3. & $10 \mathrm{~m}$ & $2 \times 10$ & Standing & $20 \times 1$ bullet & $5 \mathrm{~s}$ for one bullet \\
\hline & 4. & $10 \mathrm{~m}$ & $1 \times 5$ & Kneeling - preparatory & $1 \times 5$ bullets & unlimited \\
\hline & 5. & $7 \mathrm{~m}$ & $2 \times 5$ & Standing & $(2 \times) 5 \times 1$ bullet & $5 \mathrm{~s}$ for a bullet \\
\hline & 6. & $5 \mathrm{~m}$ & $2 \times 5$ & Standing & $5 \times 2$ bullets & $5 \mathrm{~s}$ for two bullets \\
\hline & 7. & $3 \mathrm{~m}$ & $2 \times 5$ & Standing & $5 \times 2$ bullets & $3 \mathrm{~s}$ for two bullets \\
\hline \multirow{6}{*}{ IV } & 1. & $3 \mathrm{~m}$ & $1 \times 10$ & Standing & $5 \times 2$ bullets & $3 \mathrm{~s}$ for two bullets \\
\hline & 2. & $5 \mathrm{~m}$ & $1 \times 10$ & Standing & $5 \times 2$ bullets & $4 \mathrm{~s}$ for two bullets \\
\hline & 3. & $7 \mathrm{~m}$ & $1 \times 10$ & Standing & $10 \times 1$ bullet & $4 \mathrm{~s}$ for one bullet \\
\hline & 4. & $10 \mathrm{~m}$ & $2 \times 10$ & Standing - kneeling & $2 \times 10$ bullets & $25 \mathrm{~s}$ for ten bullets \\
\hline & 5. & $10 \mathrm{~m}$ & $1 \times 15$ & Standing & $1 \times 15$ bullets & $25 \mathrm{~s}$ for fifteen bullets \\
\hline & 6. & $15 \mathrm{~m}$ & $1 \times 15$ & Standing & $1 \times 15$ bullets & $50 \mathrm{~s}$ for fifteen bullets \\
\hline \multirow[t]{2}{*}{ V } & 1. & $7 \mathrm{~m}$ & $1 \times 10$ & $\begin{array}{c}\text { Standing - preparatory } \\
\text { with a flashlight }\end{array}$ & $5 \times 2$ bullets & $5 \mathrm{~s}$ for two bullets \\
\hline & 2. & $5 \mathrm{~m}$ & $1 \times 10$ & Standing & $5 \times 2$ bullets & $4 \mathrm{~s}$ for two bullets \\
\hline
\end{tabular}

Table 3 Characteristics of the First modified training program (Prog_2)

\begin{tabular}{|c|c|c|c|c|c|c|}
\hline Series & No. & Length & Loading & Positions & Realization & Time \\
\hline \multirow{6}{*}{ I } & 1. & $5 \mathrm{~m}$ & $1 \times 10$ & Standing - preparatory & $10 \times 1$ bullet & Unlimited \\
\hline & 2. & $7 \mathrm{~m}$ & $1 \times 10$ & Standing - preparatory & $10 \times 1$ bullet & Unlimited \\
\hline & 3. & $10 \mathrm{~m}$ & $1 \times 10$ & Standing - preparatory & $10 \times 1$ bullet & Unlimited \\
\hline & 4. & $10 \mathrm{~m}$ & $1 \times 10$ & Standing - preparatory & $10 \times 1$ bullet & $5 \mathrm{~s}$ for one bullet \\
\hline & 5. & $10 \mathrm{~m}$ & $1 \times 5$ & Kneeling - preparatory & $5 \times 1$ bullet & Unlimited \\
\hline & 6. & $10 \mathrm{~m}$ & $1 \times 5$ & Kneeling - preparatory & $5 \times 1$ bullet & Unlimited \\
\hline \multirow{7}{*}{ II } & 1. & $15 \mathrm{~m}$ & $2 \times 10$ & Standing & (2x) $10 \times 1$ bullet & $8 \mathrm{~s}$ for one bullet \\
\hline & 2. & $10 \mathrm{~m}$ & $1 \times 5$ & Kneeling - preparatory & $1 \times 5$ bullets & Unlimited \\
\hline & 3. & $10 \mathrm{~m}$ & $2 \times 10$ & Standing & (2x) $10 \times 1$ bullet & $5 \mathrm{~s}$ for one bullet \\
\hline & 4. & $10 \mathrm{~m}$ & $1 \times 5$ & Kneeling - preparatory & $1 \times 5$ bullets & Unlimited \\
\hline & 5. & $7 \mathrm{~m}$ & $2 \times 5$ & Standing & $10 \times 1$ bullet & $5 \mathrm{~s}$ for a bullet \\
\hline & 6. & $5 \mathrm{~m}$ & $2 \times 5$ & Standing & $5 \times 2$ bullets & $5 \mathrm{~s}$ for two bullets \\
\hline & 7. & $3 \mathrm{~m}$ & $2 \times 5$ & Standing & $5 \times 2$ bullets & $3 \mathrm{~s}$ for two bullets \\
\hline \multirow[t]{2}{*}{ III } & 1. & $7 \mathrm{~m}$ & $1 \times 10$ & $\begin{array}{c}\text { Standing - preparatory } \\
\text { with a flashlight }\end{array}$ & $5 \times 2$ bullets & $5 \mathrm{~s}$ for two bullets \\
\hline & 2. & $5 \mathrm{~m}$ & $1 \times 10$ & Standing & $5 \times 2$ bullets & $4 \mathrm{~s}$ for two bullets \\
\hline
\end{tabular}

${ }^{1}$ A command is given for shooting each bullet, and the participants voluntarily opt for the time of shooting on their own innitiative. 
Table 4 Characteristics of the Second modified training program (Prog_3)

\begin{tabular}{ccccccc}
\hline Series & No. & Length & Loading & Positions & Realization & Time \\
\hline \multirow{4}{*}{ I } & 1. & $5 \mathrm{~m}$ & $1 \times 5$ & Standing - preparatory & $1 \times 5$ bullets & Unlimited \\
& 2. & $7 \mathrm{~m}$ & $1 \times 5$ & Standing - preparatory & $1 \times 5$ bullets & Unlimited \\
& 3. & $10 \mathrm{~m}$ & $1 \times 5$ & Standing - preparatory & $1 \times 5$ bullets & Unlimited \\
& 4. & $15 \mathrm{~m}$ & $1 \times 5$ & Standing - preparatory & $1 \times 5$ bullets & Unlimited \\
& 1. & $7 \mathrm{~m}$ & $1 \times 10$ & Standing - preparatory & $10 \times 1$ bullet & Unlimited \\
& 2. & $10 \mathrm{~m}$ & $1 \times 10$ & Standing - preparatory & $10 \times 1$ bullet & Unlimited \\
& 3. & $7 \mathrm{~m}$ & $1 \times 10$ & Standing & $10 \times 1$ bullet & 8 s for one bullet \\
II & 4. & $10 \mathrm{~m}$ & $1 \times 10$ & Standing & $5 \times 2$ bullets & 8 s for two bullets \\
& 5. & $10 \mathrm{~m}$ & $1 \times 5$ & Kneeling - preparatory & $1 \times 5$ bullets & Unlimited \\
& 6. & $10 \mathrm{~m}$ & $1 \times 5$ & Kneeling - preparatory & $1 \times 5$ bullets & Unlimited \\
& 7. & $5 \mathrm{~m}$ & $1 \times 10$ & Standing & $5 \times 2$ bullets & 4 s for two bullets \\
& 8. & $3 \mathrm{~m}$ & $1 \times 10$ & Standing & $5 \times 2$ bullets & 3 s for two bullets \\
\hline
\end{tabular}

Table 5 Characteristics of the Third modified training program (Prog_4)

\begin{tabular}{|c|c|c|c|c|c|c|}
\hline Series & No. & Length & Loading & Positions & Realization & Time \\
\hline \multirow{6}{*}{ I } & 1. & $7 \mathrm{~m}$ & $2 \times 5$ & Standing & $1 \times 10$ bullets & $35 \mathrm{~s}$ for ten bullets \\
\hline & 2. & $5 \mathrm{~m}$ & $1 \times 10$ & Standing & $5 \times 2$ bullets & $4 \mathrm{~s}$ for two bullets \\
\hline & 3. & $3 \mathrm{~m}$ & $1 \times 10$ & Standing - preparatory & $5 \times 2$ bullets & $2 \mathrm{~s}$ for two bullets \\
\hline & 4. & $10 \mathrm{~m}$ & $2 \times 5$ & Standing & $5 \times 2$ bullets & $5 \mathrm{~s}$ for two bullets \\
\hline & 5. & $10 \mathrm{~m}$ & $1 \times 10$ & Kneeling & $1 \times 10$ bullets & $45 \mathrm{~s}$ for ten bullets \\
\hline & 6. & $15 \mathrm{~m}$ & $1 \times 10$ & Standing & $1 \times 10$ bullets & Unlimited \\
\hline \multirow[t]{2}{*}{ II } & 1. & $7 \mathrm{~m}$ & $1 \times 10$ & $\begin{array}{c}\text { Standing - preparatory with } \\
\text { a flashlight }\end{array}$ & $5 \times 2$ bullets & $5 \mathrm{~s}$ for two bullets \\
\hline & 2. & $5 \mathrm{~m}$ & $1 \times 10$ & Standing & $5 \times 2$ bullets & $4 \mathrm{~s}$ for two bullets \\
\hline
\end{tabular}

Table 6 Characteristics of the Fourth modified training program (Prog_5)

\begin{tabular}{ccccccc}
\hline Series & No. & Length & Loading & Positions & Realization & Time \\
\hline \multirow{4}{*}{ I } & 1. & $5 \mathrm{~m}$ & $1 \times 10$ & Standing - preparatory & $10 \times 1$ bullet & Unlimited \\
& 2. & $7 \mathrm{~m}$ & $1 \times 10$ & Standing - preparatory & $10 \times 1$ bullet & Unlimited \\
& 3. & $7 \mathrm{~m}$ & $1 \times 10$ & Standing & $10 \times 1$ bullet & $8 \mathrm{~s}$ for one bullet \\
& 4. & $10 \mathrm{~m}$ & $1 \times 10$ & Standing & $10 \times 1$ bullet & $5 \mathrm{~s}$ for one bullet \\
\hline \multirow{4}{*}{ II } & 1. & $15 \mathrm{~m}$ & $1 \times 10$ & Standing & $1 \times 10$ bullets & unlimited \\
& 2. & $10 \mathrm{~m}$ & $1 \times 10$ & Kneeling - preparatory & $1 \times 10$ bullets & $45 \mathrm{~s}$ for ten bullets \\
& 3. & $7 \mathrm{~m}$ & $2 \times 5$ & Standing & $(2 \times 5) 1$ bullet & $5 \mathrm{~s}$ for one bullet \\
& 4. & $5 \mathrm{~m}$ & $2 \times 5$ & Standing & $5 \times 2$ bullets & $5 \mathrm{~s}$ for two bullets \\
& 5. & $3 \mathrm{~m}$ & $2 \times 5$ & Standing & $2 \times 5$ bullets & $5 \mathrm{~s}$ for two bullets \\
\hline
\end{tabular}


Table 7 Characteristics of the Fifth modified training program (Prog_6)

\begin{tabular}{|c|c|c|c|c|c|c|}
\hline Series & No. & Length & Loading & Positions & Realization & Time \\
\hline I & 1 . & $10 \mathrm{~m}$ & $10 \times 1$ & Standing - preparatory & $10 \times 1$ bullet & Unlimited \\
\hline (Air pistol) & 2. & $10 \mathrm{~m}$ & $10 \times 1$ & Standing - preparatory & $10 \times 1$ bullet & Unlimited \\
\hline \multirow{5}{*}{ II } & 1. & $15 \mathrm{~m}$ & $1 \times 10$ & Standing - preparatory & $1 \times 10$ bullets & Unlimited \\
\hline & 2. & $15 \mathrm{~m}$ & $1 \times 10$ & Standing - preparatory & $1 \times 10$ bullets & Unlimited \\
\hline & 3. & $15 \mathrm{~m}$ & $1 \times 10$ & Standing - preparatory & $1 \times 10$ bullets & Unlimited \\
\hline & 4. & $10 \mathrm{~m}$ & $1 \times 10$ & Standing - preparatory & $1 \times 10$ bullets & Unlimited \\
\hline & 5. & $7 \mathrm{~m}$ & $1 \times 10$ & Standing - preparatory & $1 \times 10$ bullets & Unlimited \\
\hline \multirow{7}{*}{ III } & 1. & $15 \mathrm{~m}$ & $1 \times 10$ & Standing - preparatory & 1 x 10 bullets & Unlimited \\
\hline & 2. & $15 \mathrm{~m}$ & $1 \times 10$ & Standing - preparatory & 1 x 10 bullets & Unlimited \\
\hline & 3. & $15 \mathrm{~m}$ & $1 \times 10$ & Standing - preparatory & $1 \times 10$ bullets & Unlimited \\
\hline & 4. & $7 \mathrm{~m}$ & $1 \times 10$ & Standing - preparatory & $1 \times 10$ bullets & Unlimited \\
\hline & 5. & $7 \mathrm{~m}$ & $1 \times 10$ & Standing - preparatory & $1 \times 10$ bullets & Unlimited \\
\hline & 6. & $7 \mathrm{~m}$ & $1 \times 10$ & Standing - preparatory & $1 \times 10$ bullets & Unlimited \\
\hline & 7. & $7 \mathrm{~m}$ & $1 \times 10$ & Standing - preparatory & $1 \times 10$ bullets & Unlimited \\
\hline \multirow{8}{*}{ IV } & 1. & $20 \mathrm{~m}$ & $1 \times 10$ & Standing - preparatory & $1 \mathrm{x} 10$ bullets & Unlimited \\
\hline & 2. & $20 \mathrm{~m}$ & $1 \times 15$ & Standing - preparatory & $1 \times 15$ bullets & Unlimited \\
\hline & 3. & $7 \mathrm{~m}$ & $1 \times 10$ & Standing - preparatory & $1 \times 10$ bullets & Unlimited \\
\hline & 4. & $7 \mathrm{~m}$ & $1 \times 10$ & Standing - preparatory & $1 \times 10$ bullets & Unlimited \\
\hline & 5. & $7 \mathrm{~m}$ & $1 \times 10$ & Standing - preparatory & $1 \times 10$ bullets & Unlimited \\
\hline & 6. & $7 \mathrm{~m}$ & $1 \times 10$ & Standing - preparatory & $1 \times 10$ bullets & Unlimited \\
\hline & 7. & $7 \mathrm{~m}$ & $1 \times 10$ & $\begin{array}{c}\text { Standing - preparatory } \\
\text { one-hand shooting stance (right) }\end{array}$ & $1 \times 10$ bullets & Unlimited \\
\hline & 8. & $7 \mathrm{~m}$ & $1 \times 10$ & $\begin{array}{c}\text { Standing - preparatory } \\
\text { one-hand shooting stance (left) }\end{array}$ & $1 \times 10$ bullets & Unlimited \\
\hline
\end{tabular}


Table 8 Characteristics of the Sixth modified training program (Prog_7)

\begin{tabular}{|c|c|c|c|c|c|c|}
\hline Series & No. & Length & Loading & Positions & Realization & Time \\
\hline I & 1. & $10 \mathrm{~m}$ & $10 \times 1$ & Standing - preparatory & $10 \times 1$ bullet & Unlimited \\
\hline (Air pistol) & 2. & $10 \mathrm{~m}$ & $10 \times 1$ & Standing - preparatory & $10 \times 1$ bullet & Unlimited \\
\hline \multirow{5}{*}{ II } & 1. & $15 \mathrm{~m}$ & $1 \times 10$ & Standing - preparatory & $1 \times 10$ bullets & Unlimited \\
\hline & 2. & $15 \mathrm{~m}$ & $1 \times 10$ & Standing - preparatory & $1 \times 10$ bullets & Unlimited \\
\hline & 3. & $15 \mathrm{~m}$ & $1 \times 10$ & Standing - preparatory & $1 \times 10$ bullets & Unlimited \\
\hline & 4. & $10 \mathrm{~m}$ & $1 \times 10$ & Standing - preparatory & $1 \times 10$ bullets & Unlimited \\
\hline & 5. & $7 \mathrm{~m}$ & $1 \times 10$ & Standing - preparatory & $1 \times 10$ bullets & Unlimited \\
\hline \multirow{7}{*}{ III } & 1. & $15 \mathrm{~m}$ & $1 \times 10$ & Standing - preparatory & $1 \times 10$ bullets & Unlimited \\
\hline & 2. & $15 \mathrm{~m}$ & $1 \times 10$ & Standing - preparatory & $1 \times 10$ bullets & Unlimited \\
\hline & 3. & $15 \mathrm{~m}$ & $1 \times 10$ & Standing - preparatory & $1 \times 10$ bullets & Unlimited \\
\hline & 4. & $7 \mathrm{~m}$ & $1 \times 10$ & Standing - preparatory & $1 \times 10$ bullets & Unlimited \\
\hline & 5. & $7 \mathrm{~m}$ & $1 \times 10$ & Standing - preparatory & $1 \times 10$ bullets & Unlimited \\
\hline & 6. & $7 \mathrm{~m}$ & $1 \times 10$ & Standing - preparatory & $1 \times 10$ bullets & Unlimited \\
\hline & 7. & $7 \mathrm{~m}$ & $1 \times 10$ & Standing - preparatory & $1 \times 10$ bullets & Unlimited \\
\hline \multirow{8}{*}{ IV } & 1. & $20 \mathrm{~m}$ & $1 \times 10$ & Standing - preparatory & $1 \times 10$ bullets & Unlimited \\
\hline & 2. & $20 \mathrm{~m}$ & $1 \times 15$ & Standing - preparatory & $1 \times 15$ bullets & Unlimited \\
\hline & 3. & $7 \mathrm{~m}$ & $1 \times 10$ & Standing - preparatory & $1 \times 10$ bullets & Unlimited \\
\hline & 4. & $7 \mathrm{~m}$ & $1 \times 10$ & Standing - preparatory & $1 \times 10$ bullets & Unlimited \\
\hline & 5. & $7 \mathrm{~m}$ & $1 \times 10$ & Standing - preparatory & $1 \times 10$ bullets & Unlimited \\
\hline & 6. & $7 \mathrm{~m}$ & $1 \times 10$ & Standing - preparatory & $1 \times 10$ bullets & Unlimited \\
\hline & 7. & $7 \mathrm{~m}$ & $1 \times 10$ & $\begin{array}{l}\text { Standing - preparatory one- } \\
\text { hand shooting stance (right) }\end{array}$ & $1 \times 10$ bullets & Unlimited \\
\hline & 8. & $7 \mathrm{~m}$ & $1 \times 10$ & $\begin{array}{l}\text { Standing - preparatory one- } \\
\text { hand shooting stance (left) }\end{array}$ & $1 \times 10$ bullets & Unlimited \\
\hline \multirow{6}{*}{ V } & 1. & $3 \mathrm{~m}$ & $1 \times 10$ & Standing - preparatory & $5 \times 2$ bullets & $2 \mathrm{~s}$ for two bullets \\
\hline & 2. & $5 \mathrm{~m}$ & $1 \times 10$ & Standing & $5 \times 2$ bullets & $4 \mathrm{~s}$ for two bullets \\
\hline & 3. & $7 \mathrm{~m}$ & $2 \times 5$ & Standing & $1 \times 10$ bullets & $35 \mathrm{~s}$ for ten bullets \\
\hline & 4. & $10 \mathrm{~m}$ & $2 \times 5$ & Standing & $5 \times 2$ bullets & $5 \mathrm{~s}$ for two bullets \\
\hline & 5. & $10 \mathrm{~m}$ & $1 \times 10$ & Kneeling & $1 \times 10$ bullets & $45 \mathrm{~s}$ for ten bullets \\
\hline & 6. & $15 \mathrm{~m}$ & $1 \times 10$ & Standing & $1 \times 10$ bullets & Unlimited \\
\hline \multirow{6}{*}{ VI } & 1. & $3 \mathrm{~m}$ & $1 \times 10$ & Standing & $5 \times 2$ bullets & $2 \mathrm{~s}$ for two bullets \\
\hline & 2. & $5 \mathrm{~m}$ & $1 \times 10$ & Standing & $5 \times 2$ bullets & $3 \mathrm{~s}$ for two bullets \\
\hline & 3. & $7 \mathrm{~m}$ & $2 \times 5$ & Standing & $1 \times 10$ bullets & $20 \mathrm{~s}$ for ten bullets \\
\hline & 4. & $10 \mathrm{~m}$ & $2 \times 5$ & Standing & $5 \times 2$ bullets & $4 \mathrm{~s}$ for two bullets \\
\hline & 5. & $10 \mathrm{~m}$ & $1 \times 10$ & Kneeling & $1 \times 10$ bullets & $20 \mathrm{~s}$ for ten bullets \\
\hline & 6. & $15 \mathrm{~m}$ & $1 \times 10$ & Standing & $1 \times 10$ bullets & $30 \mathrm{~s}$ for ten bullets \\
\hline
\end{tabular}




\section{A sample of the criterion variable}

After each completed training program, a standardized Shooting test was realized (Shoot $_{\mathrm{TEST}}$ ) (Vučković et al. 2008) for the assessment of pistol handling. The characteristics of Shoot $_{\text {TEST }}$ are shown in Table 9.

Table 9 Characteristics of the Shooting test (ShootTEST)

\begin{tabular}{ccccccc}
\hline Series & No. & Distance & Loading & Positions & Realization & Time \\
\hline \multirow{4}{*}{$\mathrm{I}$} & 1. & $3 \mathrm{~m}$ & $1 \times 10$ & Standing - preparatory & $5 \times 2$ bullets & $2 \mathrm{~s}$ for two bullets \\
& 2. & $5 \mathrm{~m}$ & $1 \times 10$ & Standing & $5 \times 2$ bullets & $4 \mathrm{~s}$ for two bullets \\
& 3. & $7 \mathrm{~m}$ & $2 \times 5$ & Standing & $1 \times 10$ bullets & $35 \mathrm{~s}$ for ten bullets \\
& 4. & $10 \mathrm{~m}$ & $2 \times 5$ & Standing & $5 \times 2$ bullets & $5 \mathrm{~s}$ for two bullets \\
& 5. & $10 \mathrm{~m}$ & $1 \times 10$ & Kneeling & $1 \times 10$ bullets & 45 s for ten bullets \\
& 6. & $15 \mathrm{~m}$ & $1 \times 10$ & Standing & $1 \times 10$ bullets & Unlimited \\
\hline
\end{tabular}

A standardized silhouette target was used for the Shoot ${ }_{\mathrm{TEST}}$, with a value of 10 only (Figure 1).

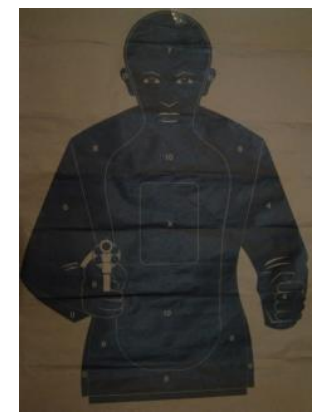

Fig 1 Silhouette target

\section{Statistical analysis}

All of the data were analyzed using the descriptive statistics to calculate the basic parameters of central tendency, the existence of a general difference of variability between the groups was determined by a One-way analysis of variance (ANOVA), while for the determination of partial difference between pairs of variables, a post hoc test with the application of Scheffe criterion was used (Hair, Anderson, Tatham \& Black, 1998; Hair, Black, Babin, Anderson \& Tatham, 2006). All statistical analyses were done by the application of software package SPSS for windows, Release 11.5.0 (Copyright by SPSS Inc., 1989-2002). 


\section{RESULTS}

The results of descriptive values for the criterion variable of the accuracy of shooting $\left(\right.$ Shoot $\left._{\mathrm{TEST}}\right)$ are shown in Table 10.

Table 10 Descriptive statistics of the shooting accuracy variable

\begin{tabular}{|c|c|c|c|c|c|c|c|c|c|}
\hline \multirow{2}{*}{ Prog. } & \multirow{2}{*}{$\mathrm{N}$} & \multirow{2}{*}{ Mean } & \multirow{2}{*}{$\mathrm{cV} \%$} & \multirow{2}{*}{ SD } & \multirow{2}{*}{$\begin{array}{l}\text { Std. } \\
\text { Error }\end{array}$} & \multicolumn{2}{|c|}{$95 \%$ Confidence Interval for Mean } & \multirow{2}{*}{ Min } & \multirow{2}{*}{ Max } \\
\hline & & & & & & Lower Bound & Upper Bound & & \\
\hline Prog_1 & 80 & 48.18 & 19.52 & 5.10 & 0.57 & 47.04 & 49.31 & 33.00 & 58.00 \\
\hline Prog_2 & 57 & 39.77 & 30.57 & 7.60 & 1.01 & 37.80 & 79 & 26.00 & 56.00 \\
\hline Prog_3 & 44 & 52.80 & 23.73 & 5.08 & 0.77 & 51 & & 39.00 & 60.00 \\
\hline Prog_4 & 56 & 51.29 & 19.83 & 5.40 & 0.72 & 4 & & 0 & 59.00 \\
\hline Prog_5 & 62 & 49.82 & 22.66 & 6.17 & 0.78 & 48.26 & 51 & 29.00 & 59.00 \\
\hline Prog_6 & 74 & 45.76 & 39.19 & 7.11 & 0.83 & 44.11 & 47.40 & 28.00 & 58.00 \\
\hline Prog_7 & 74 & 50.16 & 29.82 & 5.55 & 0.65 & 48.86 & 51.43 & 35.00 & 60.00 \\
\hline Total & 447 & 48.10 & 29.62 & 7.13 & 0.34 & 47.44 & 48.76 & 26.00 & 60.00 \\
\hline
\end{tabular}

Results of the one-way ANOVA showed that there is a statistically significant difference in shooting accuracy among the groups $(\mathrm{F}=28.840, \mathrm{p}=0.000)$.

Table 11 presents the results of Post Hoc test and Scheffe criterion that determines the existence of differences in shooting accuracy among the groups.

Table 11 Post Hoc test and Scheffe criterion

\begin{tabular}{lllllll}
\hline Prog. & Prog_1 & Prog_2 & Prog_3 & Prog_4 & Prog_5 & Prog_6 \\
\hline Prog_2 & $\mathbf{0 . 0 0 0} * * *$ & & & & & \\
Prog_3 & $\mathbf{0 . 0 1 3 *}$ & $\mathbf{0 . 0 0 0} * * *$ & & & & \\
Prog_4 & 0.198 & $\mathbf{0 . 0 0 0} * * *$ & 0.958 & & & \\
Prog_5 & 0.860 & $\mathbf{0 . 0 0 0} * * *$ & 0.408 & 0.944 & & \\
Prog_6 & 0.415 & $\mathbf{0 . 0 0 0} * * *$ & $\mathbf{0 . 0 0 0} * * *$ & $\mathbf{0 . 0 0 0} * * *$ & 0.021 & \\
Prog_7 & 0.670 & $\mathbf{0 . 0 0 0} * * *$ & 0.515 & 0.981 & 1.000 & $\mathbf{0 . 0 0 4 * *}$ \\
\hline \multicolumn{7}{c}{ * $\leq 0.05 ; * * p \leq 0.01 ; * * * p \leq 0.001$} \\
\end{tabular}

\section{DISCUSSION}

Results of the control test showed that the accuracy of shooting upon completion of different shooting programs of training was the greatest for Prog_3. The best average result of shooting accuracy was achieved in Prog_3 (52.79 \pm 5.08$)$, while the weakest was in realising Prog_2 (39.77 \pm 7.60$)$. The coefficient of variation (cV\%) shows that the groups are exceptionally homogenous in Prog_1 and Prog_4 (19.52, 19.83\%, respectively), and also in Prog_2, Prog_3, Prog_5, Prog_6 and Prog_7 (30.57, 23.73, 22.66, 39.19 and $29.82 \%$, respectively). The weakest homogeneity was expressed in Prog_6 (39.19\%), and the strongest one in Prog_1 (19.52\%). Generally observed, the results of cV\% showed that all the values presented in the shooting test belonged to homogeneous groups, so that results may be interpreted as valid in relation to the studied phenomenon.

Analysing the obtained results of the final shooting test (as the criterion variable that defines the final efficiency of the basic shooting training), it was shown that the level of 
the participants' shooting accuracy was in function of the groups: 48.18 \pm 5.10 , $39.77 \pm 7.60,52.80 \pm 5.08,51.29 \pm 5.40,49.82 \pm 6.17,45.76 \pm 7.11$ and $50.15 \pm 7.13$ points, respectively (Table 10). On the basis of the obtained results, it can be concluded that the participants who realized Prog_3 achieved the best average result on the level of accuracy of $52.80 \pm 5.08$ shots $(100 \%)$. Comparing this result with other average values, it can be concluded that the relations in percentages are the following: for Prog_1 90.21\%, Prog_2 $60.82 \%$, Prog_4 98.73\%, Prog_5 82.95\%, Prog_6 68.72\%, Prog_7 87.45\%.

ANOVA results showed that there is a statistically significant difference among the observed programs. It can be assumed that the existence of a statistically significant difference is a consequence of the influences of different training programs. On the basis of the results of Post Hoc test and Scheffe criterion, it was also concluded that among the observed training programs there were statistically significant differences. Prog_3, which appeared to be the most efficient in accuracy, was not statistically significantly different from Prog_4, Prog_5 and Prog_7.

Prog_3, Prog_6 and Prog_7 started with precise shooting in a circular target, but with pistol in Prog_3, and with air pistol at Prog_6 and Prog_7. Distances in Prog_3 were 5, 7, 10 and 15 metres, and only 10 metres in Prog_6 and Prog_7. In these programs there was no time restriction for precise shooting. The results of this study suggest that in programs that started with the use of an air pistol there is no positive transfer in accuracy of shooting with a pistol. Moreover, it can be assumed that training for pistol handling should start with precise shooting exercises with the use of a clearly defined target. The reason could be in the visual control of the shot that enabled easier control and maintaining the aiming line (Posner and Raichle, 1991).

According to the classical theory of vigilance, the reduction in alertness during the shooting is the result of the lack of stimulation (Eysenck, 2012; Finomore, Matthews, Shaw and Warm, 2009). It was confirmed that stimulation is necessary for maintaining alertness (Smit, Eling and Coenen 2004). Apart from the lack of stimulation, negative effects of monotonous tasks on performances can be additionally explained by the theory of habituation (Mackworth, 1969). The process of habituation is dynamic and, by its nature, is different from fatigue. Change in stimulation (a change in the target in case of Prog_3) may result in imminent improvement of performances, while a rest period is necessary for overcoming fatigue. In this case, stimulation and avoidance of habituation could explain the improvement of performances at frequent target changes in Prog_3. Also, as a result of practising the period of the "Quiet eye" (Janelle and Hatfield, 2008; Posner and Raichle, 1991), a frequent target change in Prog_3 has probably led to better concentration on the part of the participatns and their more quality orienting and control of visual attention. The period of "quiet eye" has been defined as a long steady duration between the final visual fixation and motor response, in this case, the beginning of triggering (Vickers, 1996). It can be concluded that the psychological aspects of changing targets, due to quality visual attention has had a positive effect on shooting training, because shooting requires intensive and focused attention (Janelle \& Hatfield, 2008).

Based on the number of fired bullets it can be concluded that the participants who fired the greatest number of bullets did not achieve the best results, which directs us to the influence of the implemented program contents. In the study that observed the influence of the applied shooting training program, the efficiency of the use of a pistol increased on the level of the constant of the first exponent amounting to $0.1094 \%$ of efficiency in the 
function of each percentile of the realized training program. When the given change in efficiency of pistol shooting is observed in the function of the bullets shot, the results have shown that with each fired bullet the efficiency grew on the level of the constant of the first exponent of $0.1406 \%$ (Vučković et al. 2008). In addition, if too many bullets are fired during training, muscle fatigue may appear and have a negative influence on shooting (Goonetilleke, Hoffmann \& Lau, 2009; Lakie, 2010; Brown, Tandy, Wulf \& Young, 2013). In general, alertness (vigilance) is the ability to sustain attention on a task during some period of time (Parasuraman, 1998). Shooting has the characteristics of a monotonous task - it is characterized by low or repetitive stimulation. During a monotonous task, cognitive and motor stimulation is reduced, which also reduces the ability of sustaining vigilance and contributes to the number of mistakes. Studies show that monotonous tasks lead to an average reduction in performances, up to $45 \%$ (a drop in performances is measured by a number of mistakes and the reduction of the time of reaction) (Larue, Rakotonirainy \& Pettitt, 2010). The reduction of alertness occurs during the first minutes of performing a monotonous task, which is not the case in non-monotonous tasks (Larue, Rakotonirainy \& Pettitt, 2009). The obtained results impose the need for the optimal number of bullets necessary for achieving the maximum effect of the training.

In Prog_ 3 the number of exercises with time limit is only 3 , that is $25 \%$ in relation to the total number of exercises (Table 1). It can be concluded that at the beginning of the training the participants should be given sufficient time for quality maintaining of the aiming line. This is the way to improve the mechanisms of kinaesthetic sense and enables the correction of the aiming line. In shooting training there are also exercises for pistol handling. In Prog_3 the number of exercises without manipulation is 8 , that is $67 \%$ of exercises of the given program. If the correctness of movements is inversely proportional to the speed of action (Barber \& Legge, 1976), it can be assumed that pistol handling training should start with exercises in which fast reactions are not dominant. When the structure of pistol manipulation movement is strong enough (drawing a pistol from the holster, loading a bullet into the firing chamber, re-inserting the magazine, aiming), the shooting training should proceed with exercises which include time limits. The reason for that is the law of cognitive structure activation, where with redistribution of sensorimotor schemes of behaviour adjustment achieves the subjective procedure with objective structure of tasks and mastering of their typical forms (Werner and Kaplan 2014). It can be concluded that the dominant exercises for precise shooting without a time limit should be at the beginning of the basic level of training, while the frequent change of targets contributes to a higher level of attention.

\section{CONCLUSIONS}

Previously, there have only been a small number of studies which evaluated the basic training in accuracy and precision in pistol use through the various shooting programs. The most probable reason is the complex organization and large costs of these trainings. Results obtained in this study may serve to choose and construct the training program in handling firearms on the basic level in various police units. Based on these results, there can be a reduction of costs of the existing training in the terms of the number of bullets and the time for training. Also, the data from this research could help in the implementation of a program which ensures the efficiency of shooting is on the desired level. 
Apart from the needs of education, such a program can also be used for the needs of assessment, control, and raising the existing levels of knowledge. In future research it is necessary to determine efficient ways of knowledge transfer from the basic to situationaldirected levels: precision and accuracy of shooting in conditions of increased levels of physical activity, in stressful situations, in conditions of reduced visibility and darkness.

It can be concluded that an eight-month-long period without organized physical activity, in this case SPE teaching, had an adverse influence on basic morphological characteristics and BMA of students, and that the results of entrance exam do not correspond to the present condition.

The recommendation that comes out of this study is that students of ACPS should be provided with organized physical activities, at least as a part of the regular curriculum, that would improve the level of their BMA or at least keep them at the level they had at the entrance exam.

Acknowledgements. This paper is the result of the research on project "Management of police organization in preventing and mitigating threats to security in the Republic of Serbia ", which is financed and carried out by the Academy of Criminalistic and Police Studies, Belgrade - the cycle of scientific projects 2015-2019.

\section{REFERENCES}

Barber, P.J., \& Legge, D. (1976). Perception and information. Essential Psychology, Methuen, London, England.

Brown, M.J., Tandy, R.D., Wulf, G., \& Young, J.C. (2013), The effect of acute exercise on pistol shooting performance of police officers, Motor Control, 17 (3), 273-282.

Burke, R.J., \& Mikkelsen, A. (2004). Burnout, job stress and attitudes towards the use of force by Norwegian police officers, Policing: An International Journal of Police Strategies \& Management, 28 (2), 269-278.

Decker, K.L., \& Huckabee, R.G. (2002). Raising the age and education requirements for police officers, Policing: An International Journal of Police Strategies \& Management, 25 (4), 789-802.

Eysenck, M. (2012). Attention and arousal: Cognition and performance. Springer Science \& Business Media, New York, U.S.A.

Finomore, V., Matthews, G., Shaw, T., \& Warm, J. (2009). Predicting vigilance: A fresh look at an old problem, Ergonomics, 52 (7), 791-808

Goonetilleke, R.S., Hoffmann, E.R., \& Lau, W.C. (2009). Pistol shooting accuracy as dependent on experience, eyes being opened and available viewing time, Applied Ergonomics, 40 (3), 500-508.

Hair, J., Anderson, R., Tatham, R., \& Black, W. (1998). Multivariate data analysis (Fifth Ed.). Prentice - Hall, Inc., U.S.A.

Hair, J., Black, B. Babin, B., Anderson, R., \& Tatham, R. (2006). Multivariate data analysis (Sixth Ed). Upper Saddle River, Prentice - Hall, Inc., U.S.A.

Hech Dominski, F., Sousa Matias, T., Elpídio Cardoso, T., Brusque Crocetta, T., \& Andrade, A. (2015). Motivação para a prática de exercícios está relacionada ao tempo de reação de policiais civis de unidades de operações especiais (Motivation for exercise is related to reaction time in police officers at special operation units), Revista Cubana de Medicina Militar, 44 (2), 195-206.

Heim, C., \& Schmidtbleicher, D. (2006). Towards an understanding of involuntary firearms discharges. Possible risks and implications for training, Policing: An International Journal of Police Strategies \& Management, 29 (3), 435-450.

Janelle, C., \& Hatfield, B. (2008). Visual attention and brain processes that underlie expert performance: Implications for sport and military psychology, Military Psychology, 20 (1), 39-69.

Kayihan, G., Ersöz, G., Özkan, A., \& Koz, M. (2013). Relationship between efficiency of pistol shooting and selected physical-physiological parameters of police, Policing: An International Journal of Police Strategies \& Management, 36 (4), 819-832. 


\section{G. VUČKOVIĆ, M. DOPSAJ, B. MITROVIĆ, R. JANKOVIĆ, S. DIMITRIJEVIĆ, S. MIJALKOVIĆ}

Lakie, M. (2010). The influence of muscle tremor on shooting performance. Experimental Physiology, 95 (3), 441-450.

Larue, G.S., Rakotonirainy, A., \& Pettitt, A.N. (2010). Real-time performance modelling of a sustained attention to response task, Ergonomics, 53 (10), 1205-1216.

Larue, G.S., Rakotonirainy, A., \& Pettitt, A.N. (2009). A model to predict hypo vigilance during a monotonous task, in Proceedings of the 2009 Australasian Road Safety Research, Policing and Education Conference: Smarter, Safer Directions, Sydney, New South Wales, Australia.

Mackworth, J.F. (1969). Vigilance and habituation: a neuropsychological approach. Harmondsworth: Penguin Books, Great Britain.

Morrison, G.B. (2005). Police department and instructor perspectives on pre-service firearm and deadly force training, Policing: An International Journal of Police Strategies \& Management, 29 (2), 226-245.

Parasuraman, R. (1998). The attentive brain. Mass.: MIT Press, Cambridge, England.

Posner, M.I.., \& Raichle, M. (1991). Images of mind, New York: Scientific, American Books, U.S.A.

Roberg, R. (2004). Higher education and policing: where are we now?, Policing: An International Journal of Police Strategies \& Management, 29 (3), 435-450.

Smit, A.S., Eling, P.A.T.M., \& Coenen, A.M.L. (2004). Mental effort causes vigilance decrease due to resource depletion, Acta Psychologica, 115 (1), 35-42.

Vickers, J.N. (1996). Visual control while aiming at a far target, Journal of Experimental Psychology: Human Perception and Performance, 22 (2), 342-354.

Vučković, G., Dopsaj, M., \& Dujković, P. (2005). Training for handling official pistol according to international standards, Science - Security - Police, 10 (3), 175-196.

Vučković, G., \& Dopsaj, M. (2007). Predicting efficiency of situational pistol shooting on the basis of motor abilities of the students of Academy of criminalistic and police studies, Serbian Journal of Sports Sciences, 1 (1), 23-36.

Vučković, G., Dopsaj, M., Radovanović, R., \& Jovanović, A. (2008). Characteristics of shooting efficiency during a basic shooting training program involving police officers of both sexes, Facta Universitatis Series Physical Education and Sport, 6 (1), 147 - 157.

Werner, H., \& Kaplan, B. (2014). Symbol formation. Psychology Press, New York, U.S.A

White, M.D. (2002). Identifying situational predictors of police shootings using multivariate analysis, Policing: An International Journal of Police Strategies \& Management, 25 (4), 726-751.

\section{EFEKTI RAZLIČITIH PROGRAMA GAĐANJA NA PRECIZNOST PIŠTOLJEM}

Upotreba vatrenog oružja - pištolja jedna je od specijalizovanih fizičkih sposobnosti policajaca $i$ važan je segment obuke u obrazovnom sistemu policije Republike Srbije, uz obavezu konstantnog praćenja postignutih rezultata. To praćenje se odnosi i na aspekt osposobljenosti $u$ rukovanju pištoljem $i$ na aspekt poboljšanja kvaliteta obuke uz smanjenje broja metaka i vremena potrebnog za realizaciju planiranih programa gađanja. Cilj studije bio je da se definiše najefikasniji i najracionalniji program obuke gađanja koji bi omogućio napredak $u$ osnovnoj obuci u rukovanju pištoljem. Uzorak je činilo 447 studenata Kriminalističko-policijske akademije, muškog pola, starosti od 19 do 22 godine, podeljenih na 7 grupa, gde je svaka grupa radila po drugačijem programu. Svi podaci su analizirani korišćenjem deskriptivne statistike, a postojanje razlike $u$ varijabilnosti na generalnom nivou između grupa utvrđivano je univarijatnom analizom varijanse (ANOVA), dok je za određivanje parcijalne razlike između parova varijabli korišćen Post-Hoc test sa primenom Scheffe-ovog kriterijuma. Rezultati su pokazali da postoji statistički značajna razlika u preciznosti gađanja između grupa $(F=28.840, p=0,000)$. Na osnovu ovih rezultata mogu se smanjiti troškovi postojeće obuke u smislu broja metaka i vremena potrebnog za obuku. Takođe, podaci iz ovog istraživanja mogli bi pomoći u realizaciji programa koji obezbeđuje efikasnost gađanja na potrebnom nivou.

Ključne reči: gađanje, pištolj, policija, obuka 